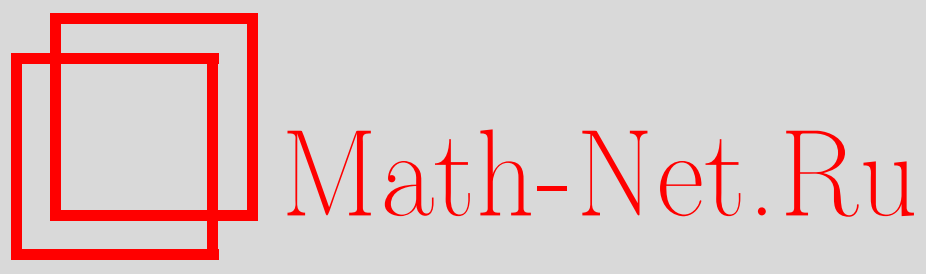

Е. В. Троицкий, Некоммутативная теорема Рисса и теорема типа Бернсайда о скрученной сопряженности, Функи. анализ и его прил., 2006, том 40, выпуск $2,44-54$

DOI: https://doi.org/10.4213/faa5

Использование Общероссийского математического портала Math-Net.Ru подразумевает, что вы прочитали и согласны с пользовательским соглашением

http://www . mathnet.ru/rus/agreement

Параметры загрузки:

IP : 34.229 .108 .108

26 апреля 2023 г., 07:21:48

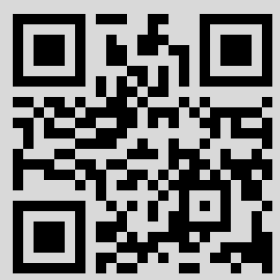


Функииональный анализ и его приложения

2006, т. 40, вып. 2, с. 44-54

УДК 517.986.66

\title{
Некоммутативная теорема Рисса и теорема типа Бернсайда о скрученной сопряженности*
}

\author{
(c) 2006. Е. В. ТРоицкий
}

\section{§1. Введение и формулировка результатов}

ОПрЕДЕЛЕНИЕ 1.1. Пусть $G$ - счетная дискретная группа, а $\phi: G \rightarrow G-$ ее эндоморфизм. Два элемента $x, x^{\prime} \in G$ называются $\phi$-сопряжсенными или скрученно-сопряженными, если имеется такое $g \in G$, что

$$
x^{\prime}=g x \phi\left(g^{-1}\right) .
$$

Мы будем обозначать через $\{x\}_{\phi}$ класс $\phi$-сопряжснности, или скрученной сопряжсенности, элемента $x \in G$. Число классов $\phi$-сопряженности называется числом Райдемайстера эндоморфизма $\phi$ и обозначается через $R(\phi)$. Если $\phi$ - тождественное отображение, то классы $\phi$-сопряженности превращаются в обычные классы сопряженности группы $G$.

Если $G$ - конечная группа, то классическая теорема Бернсайда (см., например, [2, с. 140]) утверждает, что число классов сопряженных элементов группы $G$ равно числу классов эквивалентности ее неприводимых унитарных представлений, т. е. точек унитарного дуального пространства $\widehat{G}$.

Рассмотрим автоморфизм $\phi$ конечной группы $G$. Тогда $R(\phi)$ совпадает с размерностью пространства скрученно-инвариантных функций на группе $G$, a значит, согласно теореме Петера-Вейля (о существовании двусторонне эквивариантного изоморфизма $\left.C^{*}(G) \cong \bigoplus_{\rho \in \widehat{G}} \operatorname{End}\left(H_{\rho}\right)\right)$, с суммой размерностей $d_{\rho}$ пространств скрученно-инвариантных элементов алгебр $\operatorname{End}\left(H_{\rho}\right)$, где $\rho$ пробегает $\widehat{G}$, а через $H_{\rho}$ обозначено пространство представления $\rho$. По лемме Шура $d_{\rho}=1$, если $\rho-$ неподвижная точка отображения $\hat{\phi}: \widehat{G} \rightarrow \widehat{G}, \hat{\phi}(\rho):=\rho \circ \phi$, и $d_{\rho}=0$ в противном случае. Таким образом, $R(\phi)$ совпадает с числом неподвижных точек отображения $\hat{\phi}$ (см., например, [8]). Цель данной работы - обобщить это утверждение (видоизменив его должным образом) на бесконечные дискретные группы.

ЗАмЕчАниЕ 1.2 . Если $\phi: G \rightarrow G-$ эпиморфизм, то он индуцирует отображение $\hat{\phi}: \widehat{G} \rightarrow \widehat{G}, \hat{\phi}(\rho)=\rho \circ \phi$ (поскольку представление неприводимо тогда и только тогда, когда лишь скалярные операторы в пространстве представления коммутируют со всеми операторами представления). Это не так для произвольного эндоморфизма $\phi$, поскольку $\rho \circ \phi$ может быть приводимым для неприводимого представления $\rho$ и $\hat{\phi}$ можно определить только как многозначное отображение. Тем не менее можно следующим образом определить множество

* Работа частично поддержана грантом РФФИ 05-01-00923, грантом Поддержки ведущих научных школ НШ-619.2003.1 и грантом «Университеты России» УР.04.02.530. 
неподвижных точек $\operatorname{Fix}(\hat{\phi})$ отображения $\hat{\phi}$ на $\widehat{G}$ для случая общего эндоморфизма.

ОПРЕДЕЛЕНИЕ 1.3. Пусть $\operatorname{Rep}(G)$ - множество классов эквивалентности конечномерных унитарных представлений группы $G$. Тогда соответствующее отображение $\hat{\phi}_{R}: \operatorname{Rep}(G) \rightarrow \operatorname{Rep}(G)$ определяется, как и выше, по формуле $\hat{\phi}_{R}(\rho)=\rho \circ \phi$.

Обозначим через $\operatorname{Fix}(\hat{\phi})$ множество таких точек $\rho \in \widehat{G} \subset \operatorname{Rep}(G)$, что $\hat{\phi}_{R}(\rho)$ $=\rho$.

TЕоремА 1.4 (сильная теорема типа Бернсайда для групп типа I [9]). Пусть $G$ - конечно порожденная дискретная группа типа I, $\phi$ - ее автоморбизм, $R(\phi)$ - число классов $\phi$-сопряжснности, a $S(\phi)=\# \operatorname{Fix}(\hat{\phi})$ - число $\hat{\phi}$-инвариантных классов неприводимых унитарных представлений группы $G$. Eсли одно из чисел $R(\phi)$ и $S(\phi)$ конечно, то оно равняется другому.

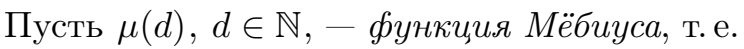

$$
\mu(d)= \begin{cases}1, & \text { если } d=1, \\ (-1)^{k}, & \text { если } d-\text { произведение } k \text { различных простых чисел, } \\ 0, & \text { если } d \text { не свободно от квадратов. }\end{cases}
$$

Теорема 1.5 (сравнения для чисел Райдемайстера [9]). Пусть $\phi: G \rightarrow G-$ такой эндоморфизм счетной дискретной группы $G$, что все числа $R\left(\phi^{n}\right)$ конечны, и пусть $H$ - подгруппа в $G$ со следующими свойствами: $\phi(H) \subset H u$ для всякого $x \in G$ найдется такое $n \in \mathbb{N}$, что $\phi^{n}(x) \in H$. Eсли пара $\left(H, \phi^{n}\right)$ удовлетворяет условиям теоремы 1.4 для любого $n \in \mathbb{N}$, то для любого $n$ выполняется соотношение

$$
\sum_{d \mid n} \mu(d) \cdot R\left(\phi^{n / d}\right) \equiv 0 \bmod n .
$$

Для групп типа $\mathrm{II}_{1}$ ситуация гораздо сложнее. Так, например, в случае полупрямого произведения по действию группы $\mathbb{Z}$ на $\mathbb{Z} \oplus \mathbb{Z}$ гиперболическим автоморфизмом в [10] найден такой автоморфизм $\phi$, что его число Райдемайстера конечно (а именно, равно четырем), а число неподвижных точек отображения $\hat{\phi}$ на $\widehat{G}$ больше или равно пяти. Это явление связано с плохими свойствами отделимости пространства $\widehat{G}$ для общих дискретных групп. Более глубокое изучение ситуации приводит к следующей общей теореме, составляющей один из двух основных результатов данной работы.

ТЕорема 1.6 (слабая теорема типа Бернсайда для скрученных классов). Размерность $R_{*}(\phi)$ пространства скрученно-инвариантных функиий на $G$, лежащих в алгебре Фуръе-Стилтвеса $B(G)$, равна числу $S_{*}(\phi)$ обобщенных неподвижных точек I гомеоморфизма $\hat{\phi}$ (сумме коразмерностей пространств, порожденных элементами вида $a-\delta_{g} * a * \delta_{\phi\left(g^{-1}\right)}+I$ в $C^{*}(G) / I$, где $\delta_{g}-$ делътафункция с носителем в $g$ ) на спектре Глимма группь $G$, m. е. на полной регуляризации пространства $\widehat{G}$, если хотя бъ одно из чисел $R_{*}(\phi)$ и $S_{*}(\phi)$ конечно.

Этот результат во многих случаях позволяет получить сильную форму теоремы типа Бернсайда $R(\phi)=S(\phi)$. В доказательстве обобщенной теоремы Бернсайда в [9] для групп типа I (см. теорему 1.4 данной работы, а также [8, 10]) 
использовано отождествление $R(\phi)$ и размерности пространства скрученноинвариантных $\left(L^{\infty}\right.$-)функций на $G$, т. е. скрученно-инвариантных функционалов на $L^{1}(G)$. Поскольку только часть $L^{\infty}$-функций определяет функционалы на $C^{*}(G)$ (а именно, функции Фурье-Стилтьеса), то a priori имеем $R_{*}(\phi) \leqslant R(\phi)$. Тем не менее функции с некоторыми условиями симметрии очень часто лежат в алгебре Фурье-Стилтьеса, так что можно высказать гипотезу о том, что $R(\phi)=R_{*}(\phi)$, если $R(\phi)<\infty$. Так происходит во всех известных примерах.

Слабая теорема будет доказана следующим образом. Хорошо известная теорема Рисса(-Маркова-Какутани) отождествляет пространство линейных функционалов на алгебре $A=C(X)$ и пространство регулярных мер на $X$. Для доказательства слабой теоремы типа Бернсайда мы сначала получаем обобщение теоремы Рисса на случай некоммутативной $C^{*}$-алгебры $A$ при помощи теоремы Даунса-Хофманна о представлении элементов алгебры сечениями. Это - второй основной результат данной работы. В нашем случае соответствующие меры на спектре Глимма являются мерами со значениями в функционалах. В коммутативной ситуации и в случае одноточечного спектра Глимма эта теорема либо сводится к теореме Рисса, либо вырождается в тавтологию, однако для групповых $C^{*}$-алгебр дискретных групп во многих случаях получается новый инструмент для подсчета классов скрученной сопряженности.

Интерес к отношениям скрученной сопряженности берет начало, в частности, в теории неподвижных точек Нильсена-Райдемайстера (см., например, [12, 8]), в теории Сельберга (см., например, [14,5]) и алгебраической геометрии (см., например, [11]). Заметим, что число Райдемайстера эндоморфизма конечно порожденной абелевой группы бесконечно тогда и только тогда, когда 1 не принадлежит спектру ограничения этого эндоморфизма на свободную часть группы (см., например, [12]). Число Райдемайстера бесконечно для любого автоморфизма неэлементарной гиперболической по Громову группы [4].

Настоящая работа представляет собой часть исследовательской программы, выполняемой в Институте математики Макса Планка (MPI) в Бонне совместно с А. Фельштыном. Автор выражает благодарность этому институту за поддержку и гостеприимство в течение того времени, когда была получена большая часть результатов настоящей работы, а также MPI и организаторам рабочих встреч по некоммутативной геометрии и теории чисел I, II (Бонн, август 2003 и июнь 2004), где была доложена значительная часть этих результатов.

Автор благодарен М. Б. Бекка, Л. И. Вайнерману, А. М. Вершику, В. М. Мануйлову, А. С. Мищенко, А. Л. Фельштыну, А. И. Штерну за полезные обсуждения, а также рецензенту за ценные замечания.

\section{§2. Алгебры операторных полей}

Прежде всего, следуя [7] (см. также [1, §10]), напомним некоторые сведения из теории операторных полей. Пусть $T$ - топологическое пространство, для каждой точки $t \in T$ которого зафиксирована $C^{*}$-алгебра (более общо - инволютивная банахова алгебра) $A_{t}$.

ОПРЕДЕЛЕНИЕ 2.1. Структурой непреръвности для $T$ и семейства $\left\{A_{t}\right\}$ называется линейное пространство $F$ операторных полей на $T$ со значениями в $\left\{A_{t}\right\}$ (т. е. отображений, переводящих $t \in T$ в некоторый элемент алгебры $A_{t}$ ), обладающее следующими свойствами: 
(1) если $x \in F$, то вещественнозначная функция $t \mapsto\|x(t)\|$ непрерывна на $T$;

(2) для каждого $t \in T$ множество $\{x(t) \mid x \in F\}$ плотно в $A_{t}$;

(3) $F$ замкнуто относительно поточечного умножения и инволюции.

ОПРЕДЕЛЕНИЕ 2.2. Операторное поле $x$ называется непрерывным по отношению к $F$ в точке $t_{0}$, если для каждого $\varepsilon>0$ найдется такой элемент $y \in F$ и такая окрестность $U$ точки $t_{0}$, что $\|x(t)-y(t)\|<\varepsilon$ для всех $t \in U$. Поле $x$ непрерывно на $T$, если оно непрерывно во всех точках этого пространства.

ОПРЕДЕЛЕНИЕ 2.3. Полная алгебра операторных полей - это семейство $A$ операторных полей на $T$, обладающее следующими свойствами:

(1) $A$ является *-алгеброй, т. е. замкнуто относительно поточечных алгебраических операций;

(2) для каждого $x \in A$ функция $t \mapsto\|x(t)\|$ непрерывна на $T$ и обращается в нуль на бесконечности;

(3) для каждого $t$ множество $\{x(t) \mid x \in A\}$ плотно в $A_{t}$;

(4) $A$ полно по норме $\|x\|=\sup _{t}\|x(t)\|$.

Полная алгебра операторных полей очевидным образом является структурой непрерывности. Если $F$ - произвольная структура непрерывности, то пусть $C_{0}(F)$ - семейство всех тех операторных полей $x$, которые непрерывны на $T$ по отношению к $F$ и для которых функции $t \mapsto\|x\|$ обращаются в нуль на бесконечности. Можно показать, что $C_{0}(F)$ - полная алгебра операторных полей.

ЛЕмма 2.4. Для любой полной алгебры $А$ операторных полей на $T$ следующие условия эквивалентны:

(1) $A$ - максимальная полная алгебра операторных полей;

(2) $A=C_{0}(F)$ для некоторой структурь непрерывности $F$;

(3) $A=C_{0}(A)$.

Такая максимальная полная алгебра $A$ операторных полей иногда называется непрерывной прямой суммой семейства $\left\{A_{t}\right\}$. Мы будем изучать унитальный случай; при этом пространство $T$ будет компактным и условия обнуления на бесконечности для сечений не будет. Более того, мы будем предполагать, что $T$ хаусдорфово, а значит, нормально. Полная алгебра в этом случае, очевидно, является разделяющей в том смысле, что если $s, t \in T, s \neq t, \alpha \in A_{s}, \beta \in A_{t}$, то найдется такой элемент $x \in A$, что $x(s)=\alpha, x(t)=\beta$. Нам понадобится различать алгебру $A$ и ее же, реализованную как алгебру сечений $\Gamma(\mathscr{A})$ поля алгебр $\mathscr{A}=\left\{A_{t}\right\}$. Мы будем обозначать через $\hat{a} \in \Gamma(\mathscr{A})$ сечение, соответствующее элементу $a \in A$.

\section{§3. Функционалы и меры}

ОПредЕЛЕниЕ 3.1. Пусть $\Sigma$ - некоторая алгебра подмножеств пространства T. Мера, связанная с максимальной полной алгеброй операторных полей $A=\Gamma(\mathscr{A})$, - это функция множеств $\mu: S \mapsto \mu(S) \in \Gamma(\mathscr{A})^{*}=A^{*}$, где $S \in \Sigma$, причем $\mu(S)(a)=0$, если supp $\hat{a} \cap S=\varnothing$. Мы будем использовать для этой меры сокращение МАОП.

Она аддитивна, если $\mu\left(\bigsqcup S_{i}\right)(a)=\sum_{i} \mu\left(S_{i}\right)(a)$.

Эта мера ограничена, если верхняя грань суммы $\sum_{i}\left\|\mu\left(S_{i}\right)\right\|$ по разбиениям $\left\{S_{i}\right\}$ пространства $T$ конечна. Эта верхняя грань обозначается через $\|\mu\|$.

Ограниченная аддитивная МАОП будет сокращенно называться ОА МАОП. 
ОПРЕДЕЛЕНИЕ 3.2. ОА МАОП назЫвается *-слабо регулярной (РОА МАОП), если для любых $E \in \Sigma, a \in A$ и $\varepsilon>0$ найдутся множество $F \in \Sigma$, замыкание которого содержится в $E$, и множество $G \in \Sigma$, внутренность которого содержит $E$, такие, что $|\mu(C) a|<\varepsilon$ для любого $C \in \Sigma$, удовлетворяющего условию $C \subset$ $G \backslash F$.

В дальнейшем в качестве $\Sigma$ мы в одном случае рассмотрим алгебру всех подмножеств пространства $T$, а в другом - алгебру, порожденную его замкнутыми подмножествами.

ОПРЕДЕЛЕНИЕ 3.3. Пусть МАОП $\lambda$ определена на алгебре $\Sigma$ подмножеств пространства $T$, причем $\lambda(\varnothing)=0$. Множество $E \in \Sigma$ называется $\lambda$-множеством, если для любого $M \in \Sigma$

$$
\lambda(M)=\lambda(M \cap E)+\lambda(M \cap(T \backslash E)) .
$$

Лемма 3.4. Пусть $\lambda$ есть МАОП, определенная на алгебре $\Sigma$ подмножеств в $T$, причем $\lambda(\varnothing)=0$. Семейство $\lambda$-множеств является подалгеброй в $\Sigma$, на которой $\lambda$ аддитивна. Более того, если $E$ - обгединение конечного набора $\left\{E_{n}\right\}$ дизбюнктных $\lambda$-множеств и $M \in \Sigma$, то $\lambda(M \cap E)=\sum_{n} \lambda\left(M \cap E_{n}\right)$.

ДокАЗАТЕЛЬСтво. Ясно, что пустое множество, все пространство и дополнение к любому $\lambda$-множеству являются $\lambda$-множествами. Пусть теперь $X$ и $Y$ суть $\lambda$-множества, а $M \in \Sigma$. Тогда, поскольку $X-$ это $\lambda$-множество,

$$
\lambda(M \cap Y)=\lambda(M \cap Y \cap X)+\lambda(M \cap Y \cap(T \backslash X)),
$$

а поскольку $Y$ есть $\lambda$-множество,

$$
\begin{aligned}
\lambda(M)= & \lambda(M \cap Y)+\lambda(M \cap(T \backslash Y)), \\
\lambda(M \cap(T \backslash(X \cap Y)))= & \lambda(M \cap(T \backslash(X \cap Y)) \cap Y) \\
& +\lambda(M \cap(T \backslash(X \cap Y)) \cap(T \backslash Y)) ;
\end{aligned}
$$

следовательно,

$$
\lambda(M \cap(T \backslash(X \cap Y)))=\lambda(M \cap(T \backslash X) \cap Y)+\lambda(M \cap(T \backslash Y)) .
$$

Из (1) и (2) вытекает, что

$$
\lambda(M)=\lambda(M \cap Y \cap X)+\lambda(M \cap Y \cap(T \backslash X))+\lambda(M \cap(T \backslash Y)),
$$

а из (3) - что

$$
\lambda(M)=\lambda(M \cap Y \cap X)+\lambda(M \cap(T \backslash(X \cap Y))) .
$$

Таким образом, $X \cap Y$ является $\lambda$-множеством. Поскольку $\bigcup X_{n}=T \backslash \bigcap\left(T \backslash X_{n}\right)$, получаем, что $\lambda$-множества образуют алгебру. Если теперь $E_{1}$ и $E_{2}$ - дизъюнктные $\lambda$-множества, то, заменяя $M$ на $M \cap\left(E_{1} \cup E_{2}\right)$ в определении 3.3 , получаем, что

$$
\lambda\left(M \cap\left(E_{1} \cup E_{2}\right)\right)=\lambda\left(M \cap E_{1}\right)+\lambda\left(M \cap E_{2}\right) .
$$

Последнее утверждение леммы получается отсюда по индукции.

Как известно, любой функционал $\tau$ на $C^{*}$-алгебре $B$ представляется в виде линейной комбинации четырех положительных функционалов следующим 
каноническим способом. Сначала представляем $\tau$ в виде $\tau=\tau_{1}+i \tau_{2}$, где самосопряженные функционалы $\tau_{1}$ и $\tau_{2}$ определены формулами

$$
\tau_{1}(a)=\frac{\tau(a)+\overline{\tau\left(a^{*}\right)}}{2}, \quad \tau_{2}(a)=\frac{\tau(a)-\overline{\tau\left(a^{*}\right)}}{2 i} .
$$

По лемме о разложении Жордана каждый самосопряженный функционал $\alpha$ однозначно представляется в виде разности $\alpha_{+}-\alpha_{-}$двух положительных функционалов, если потребовать, чтобы

$$
\|\alpha\|=\left\|\alpha_{+}\right\|+\left\|\alpha_{-}\right\|
$$

(см. [3, $\S 3.3 ; 13$, Theorem 3.2.5]). Разложим соответствующим образом и МАОП. В силу однозначности разложения слагаемые будут МАОП. Если же мы имеем ОА МАОП, то аддитивность будет следовать из однозначности, а ограниченность (с удвоенной константой) - из формул (4) и условия (5). По тем же соображениям слагаемые будут *-слабо регулярны, если исходная МАОП являлась таковой. Таким образом, МАОП, фигурирующие в разложении, будут положительными в том смысле, что

$$
\mu(E)\left(a^{*} a\right) \geqslant 0
$$

для любого $E \in \Sigma$. В частности, они являются неубывающими по отношению к вложению множеств.

ЛЕмма 3.5. Множества $F$ и $G$ в определении 3.2 могут быть выбраны таким образом, что $|\mu(C)(f a)|<\varepsilon$ для любой непрерывной функиии $f: T \rightarrow$ $[0,1]$.

Доказательство. Рассмотрим разложения $\mu=\sum_{i=1}^{4} x_{i} \mu_{i}, a=\sum_{j=1}^{4} y_{j} a_{j}$, где $\mu_{i}$ и $a_{j}$ положительны, а $x_{i}, y_{j}$ - комплексные числа модуля $\leqslant 1$. Согласно определению 3.2 , выберем множества $F$ и $G$ для $\varepsilon / 16$, причем одновременно для всех пар $\mu_{i}, a_{j}$. Тогда

$$
0 \leqslant \mu_{i}(C)\left(f \cdot a_{j}\right)=\mu_{i}(C)\left(\left(a_{j}\right)^{1 / 2} f\left(a_{j}\right)^{1 / 2}\right) \leqslant \mu_{i}(C)\left(a_{j}\right) \leqslant \frac{\varepsilon}{16}
$$

И

$$
\left|\mu_{i}(C)(f \cdot a)\right| \leqslant \sum_{i, j=1}^{4}\left|x_{i} y_{j}\right| \cdot\left|\mu_{i}(C)\left(a_{j}\right)\right| \leqslant 16 \cdot \frac{\varepsilon}{16}=\varepsilon .
$$

ТЕОрема 3.6. Пусть сепарабельная унитальная $C^{*}$-алгебра $А$ изоморфна полной алгебре операторных полей $Г(\mathscr{A})$ над хаусдорбовым пространством $T$. Тогда функииональ на $A \cong \Gamma(\mathscr{A})$ отождествляются с РОА МАОП, связанными с $\Gamma(\mathscr{A})$.

ДокАЗАтЕЛьство. Заметим, что из условий теоремы следует, что $T$ - сепарабельный хаусдорфов компакт, а единичный шар сопряженного к $A$ пространства в *-слабой топологии - метризуемый компакт. Очевидно, РОА МАОП образуют линейное нормированное пространство относительно $\|\cdot\|$.

Прежде всего, мы хотим доказать, что естественное линейное отображение $\mu \longmapsto \mu(T)$ является изометрией пространства, состоящего из РОА МАОП, в $A^{*}$. Поскольку $\|\mu(T)\| \leqslant\|\mu\|$, то его норма не превосходит единицы. Выберем теперь 
сколь угодно малое $\varepsilon>0$. Пусть $E_{1}, \ldots, E_{n}$ - такое разбиение пространства $T$, чTо

$$
\sum_{i=1}^{n}\left\|\mu\left(E_{i}\right)\right\| \geqslant\|\mu\|-\varepsilon .
$$

Пусть $a_{i} \in A$ - такие элементы нормы 1 , что $\mu\left(E_{i}\right)\left(a_{i}\right) \geqslant\left\|\mu\left(E_{i}\right)\right\|-\varepsilon / n$.

В силу *-слабой регулярности $\mu$ и нормальности пространства $T$ можно выбрать такие замкнутые множества $C_{i}$, дизъюнктные открытые множества $G_{i}$ и непрерывные функции $f_{i}: T \rightarrow[0,1]$, что $C_{i} \subset E_{i},\left|\mu\left(E_{i} \backslash C_{i}\right)\left(a_{j}\right)\right| \leqslant \varepsilon / n^{2}$, $C_{i} \subset G_{i},\left|\mu\left(G_{i} \backslash C_{i}\right)\left(a_{j}\right)\right| \leqslant \varepsilon / n^{2}$ (причем оценки остаются верными и после умножения на положительные функции, как в лемме 3.5$), f_{i}(s)=0$, если $s \notin G_{i}$, и $f_{i}(s)=1$, если $s \in C_{i}, i, j=1, \ldots, n$.

Рассмотрим элемент вида $a:=\sum_{i} f_{i} a_{i} \in \Gamma(\mathscr{A})=A$. Тогда $\|a\| \leqslant 1$ и

$$
\begin{aligned}
|\mu(S)(a)-\|\mu\|| & \leqslant \sum_{i=1}^{n}\left|\mu\left(E_{i}\right)(a)-\mu\left(E_{i}\right)\right|+\varepsilon \\
& \leqslant \sum_{i=1}^{n}\left|\mu\left(E_{i} \backslash C_{i}\right)(a)+\mu\left(C_{i}\right)(a)-\mu\left(E_{i}\right)\left(a_{i}\right)\right|+2 \varepsilon \\
& =\sum_{i=1}^{n}\left|\sum_{j=1}^{n} \mu\left(E_{i} \backslash C_{i}\right)\left(f_{j} a_{j}\right)+\mu\left(C_{i}\right)\left(a_{i}\right)-\mu\left(E_{i}\right)\left(a_{i}\right)\right|+2 \varepsilon \\
& \leqslant \sum_{i, j=1}^{n}\left|\mu\left(E_{i} \backslash C_{i}\right)\left(f_{j} a_{j}\right)\right|+\sum_{i=1}^{n}\left|\mu\left(E_{i} \backslash C_{i}\right)\left(a_{i}\right)\right|+2 \varepsilon \\
& \leqslant n^{2} \frac{\varepsilon}{n^{2}}+n \frac{\varepsilon}{n^{2}}+2 \varepsilon \leqslant 4 \varepsilon .
\end{aligned}
$$

Поскольку $\varepsilon$ сколь угодно мало, $\|\mu\|=\|\mu(S)\|$.

Остается представить произвольный функционал $\varphi$ как РОА МАОП. Этот функционал на $Г(\mathscr{A})$ может быть продолжен по теореме Хана-Банаха до непрерывного функционала $\psi$ на $B(\mathscr{A})=\prod_{t \in T} A_{t}$ (т. е. $C^{*}$-алгебре не обязательно непрерывных сечений поля $\mathscr{A}$ c sup-нормой). Этот функционал разлагается в сумму $\psi=\sum_{i=1}^{4} \alpha_{i} \psi_{i}$, где $\psi_{i}$ - положительные функционалы, $\alpha_{i} \in \mathbb{C},\left|\alpha_{i}\right|=1$, $\left\|\psi_{i}\right\| \leqslant\|\psi\|$ (см. выше). Положим

$$
\lambda(E)(a):=\psi\left(\chi_{E} a\right), \quad \lambda_{i}(a):=\psi_{i}\left(\chi_{E} a\right), \quad i=1, \ldots, 4,
$$

где $a \in \Gamma(\mathscr{A})$, а $\chi_{E}$ - характеристическая функция множества $E$. Очевидно, что $\lambda(T)(a)=\psi(a)$ и $\lambda$ является ОА МАОП. В самом деле, первые два свойства из определения 3.1 очевидны. Третье проверяется для каждой меры $\lambda_{i}, i=$ $1, \ldots, 4$ :

$$
\sum_{j=1}^{N}\left|\lambda_{i}\left(E_{j}\right)\right|=\sum_{j=1}^{N} \lambda_{i}\left(E_{j}\right)(\mathbf{1})=\sum_{j=1}^{N} \psi_{i}\left(\chi_{E_{j}} \mathbf{1}\right)=\psi_{i}(\mathbf{1}) \leqslant\left\|\psi_{i}\right\|
$$

а значит,

$$
\sum_{j=1}^{N}\left|\lambda\left(E_{j}\right)\right| \leqslant \sum_{i=1}^{4} \sum_{j=1}^{N}\left|\lambda_{i}\left(E_{j}\right)\right| \leqslant \sum_{i=1}^{4}\left\|\psi_{i}\right\| \leqslant 4 \cdot\|\psi\| .
$$


Теперь нам надо найти такую РОА МАОП $\mu$, что $\mu(T)(a)=\lambda(T)(a)$. Без ограничения общности это достаточно сделать для положительной меры $\lambda=\lambda_{i}$.

Пусть в пространстве $T$ через $F$ обозначается произвольное замкнутое подмножество, через $G$ - произвольное открытое подмножество и через $E-$ произвольное подмножество. Положим

$$
\mu_{1}(F)\left(a^{*} a\right)=\inf _{G \supset F} \lambda(G)\left(a^{*} a\right), \quad \mu_{2}(E)\left(a^{*} a\right)=\sup _{F \subset E} \mu_{1}(F)\left(a^{*} a\right)
$$

и затем продолжим эти функционалы по линейности. Точнее, в силу сепарабельности пространства $T$ можно выбрать конфинальную последовательность $\left\{G_{i}\right\}$, такую, что $G_{i} \supset F$. Поскольку единичный шар сопряженного пространства слабо компактен, то можно выбрать слабо сходящуюся подпоследовательность функционалов $\lambda\left(G_{i_{k}}\right)$. Ее предел $\psi$ - положительный функционал на $A$, обладающий требуемым inf-свойством на положительных элементах. В частности, он не зависит от выбора $\left\{G_{i}\right\}$ и $\left\{G_{i_{k}}\right\}$. Аналогично строится $\mu_{2}$.

Эти функции множеств $\mu_{1}$ и $\mu_{2}$ неотрицательные и неубывающие. Пусть $G_{1}$ - открытое, а $F_{1}$ - замкнутое множества. Тогда если $G \supset F_{1} \backslash G_{1}$, то $G_{1} \cap G \supset F_{1}$ и $\lambda\left(G_{1}\right) \leqslant \lambda\left(G_{1}\right)+\lambda(G)$, так что $\mu_{1}\left(F_{1}\right) \leqslant \lambda\left(G_{1}\right)+\lambda(G)$. Поскольку $G$ - произвольное открытое множество, содержащее $F_{1} \backslash G_{1}$, мы получаем

$$
\mu_{1}\left(F_{1}\right) \leqslant \lambda\left(G_{1}\right)+\mu_{1}\left(F_{1} \backslash G_{1}\right) .
$$

Если $F$ - замкнутое множество, то из этого неравенства, заставляя $G_{1}$ пробегать все открытые множества, содержащие $F \cap F_{1}$, получаем, что

$$
\mu_{1}\left(F_{1}\right) \leqslant \mu_{1}\left(F \cap F_{1}\right)+\mu_{2}\left(F_{1} \backslash F\right) .
$$

Если $E-$ произвольное подмножество в $T$ и $F_{1}$ пробегает замкнутые подмножества множества $E$, то из предыдущего неравенства следует, что

$$
\mu_{2}(E) \leqslant \mu_{2}(E \cap F)+\mu_{2}(E \backslash F) .
$$

Далее мы покажем, что для произвольного множества $E$ и произвольного замкнутого множества $F$ в $T$ выполнено неравенство

$$
\mu_{2}(E) \geqslant \mu_{2}(E \cap F)+\mu_{2}(E \backslash F) .
$$

Чтобы убедиться в этом, обозначим через $F_{1}$ и $F_{2}$ дизъюнктные замкнутые множества. Поскольку $T$ нормально, имеются дизъюнктные окрестности $G_{1}$ и $G_{2}$ множеств $F_{1}$ и $F_{2}$ соответственно. Если $G$ - произвольная окрестность множества $F_{1} \cup F_{2}$, то $\lambda(G) \geqslant \lambda\left(G \cap G_{1}\right)+\lambda\left(G \cap G_{2}\right)$, так что

$$
\mu_{1}\left(F_{1} \cap F_{2}\right) \geqslant \mu_{1}\left(F_{1}\right)+\mu_{2}\left(F_{2}\right) .
$$

Пусть теперь $E$ и $F$ - произвольные множества в $T$, причем $F$ замкнуто, и пусть $F_{1}$ пробегает замкнутые подмножества в $E \cap F$, а $F_{2}$ - замкнутые подмножества в $E \backslash F$. Тогда предыдущее неравенство доказывает (7). Из (6) и (7) получаем

$$
\mu_{2}(E)=\mu_{2}(E \cap F)+\mu_{2}(E \cap(T \backslash F))
$$

для любого подмножества $E$ в $T$ и замкнутого подмножества $F$. Функция $\mu_{2}$ определена на алгебре всех подмножеств пространства $T$, и, как следует из (8), любое замкнутое множество $F$ является $\mu_{2}$-подмножеством. Если $\mu$ - ограничение меры $\mu_{2}$ на алгебру, порожденную замкнутыми множествами, то из леммы 3.4 следует, что $\mu$ аддитивна на этой алгебре. Из определений мер $\mu_{1}$ и $\mu_{2}$ ясно, что $\mu_{1}(F)=\mu_{2}(F)=\mu(F)$, если $F$ замкнуто, и, значит, $\mu(E)=\sup _{F \subset E} \mu(F)$. 
Это показывает, что $\mu$ *-слабо регулярна и, так как $\|\mu(T)\|<\infty$, то $\mu$ есть РОА МАОП.

Наконец, по определению $\mu(S)(a)=\lambda(S)(a)=\psi(a)=\varphi(a)$ для всех $a \in$ $\Gamma(\mathscr{A})$.

\section{§4. Скрученно-инвариантные МАОП}

Большая часть следующих рассуждений верна для различных представлений алгебр операторными полями, но мы ограничимся теперь случаем групповой $C^{*}$-алгебры дискретной группы и сосредоточимся на следующем важном представлении сечениями, принадлежащем Даунсу и Хофманну [6, Corollaries $8.13,8.14]$.

Пусть $Z$ - центр $C^{*}$-алгебры $A$, а $\widehat{Z}$ - пространство его максимальных идеалов, снабженное обычной топологией. Если $I \in \mathscr{P}:=\operatorname{Prim}(A)$ (пространство ядер неприводимых унитарных представлений), то $Z \cap I \in \widehat{Z}$ (это следует из того, что если взять неприводимое представление с ядром $I$, то его ограничение на $Z$ задает гомоморфизм $Z \rightarrow \mathbb{C}$, а следовательно, $Z \cap I-$ максимальный идеал в $Z$ ). Получаем отображение $f: \mathscr{P} \rightarrow \widehat{Z}$. Положим $T=f(\mathscr{P})$. Для каждого $x \in T$ рассмотрим идеал $I_{x}:=\bigcap I, f(I)=x$ (uдеал Глимма), и поле алгебр $A / I_{x}$. Возникает отображение $a \mapsto\left\{x \mapsto a+I_{x}\right\}$ из алгебры $A$ в алгебру сечений указанного поля. Важный результат, полученный в работе [6], состоит в том, что это отображение является изоморфизмом. При этом отображение $f: \mathscr{P} \rightarrow T$ универсально по отношению к непрерывным отображениям $g: \mathscr{P} \rightarrow S$ в хаусдорфовы пространства, т. е. любое такое отображение $g$ можно представить в виде $h \circ f$ для некоторого непрерывного $h: T \rightarrow S$. В случае унитальной алгебры пространство $T$ компактно.

Теперь рассмотрим счетную дискретную группу $G$ и ее автоморфизм $\phi$. Пусть $A=C^{*}(G)$. Рассмотрим скрученное действие группы $G$ на $A$

$$
g[a]:=\delta_{g} * a * \delta_{\phi\left(g^{-1}\right)},
$$

где $\delta_{g}$ - дельта-функция с носителем в $g$, и аналогичное действие на функционалах, поскольку они реализуются как некоторые функции на $G$ (оно совпадает с сопряженным действием с точностью до замены $g$ на $g^{-1}$ ). Это же действие определено и на $A / I$, поскольку идеалы инвариантны относительно сдвигов.

ОПРЕДЕЛЕНИЕ 4.1. Размерность $R_{*}(\phi)$ пространства скрученно-инвариантных функционалов на $C^{*}(G)$ называется обобщенным числом Райдемайстера автоморфизма $\phi$. Таким образом, $R_{*}(\phi)$ является размерностью пространства скрученно-инвариантных элементов алгебры Фурье-Стилтьеса $B(G)$.

Напомним, что алгебра Фурье-Стилтьеса дискретной группы $G$ определяется одним из трех эквивалентных способов (см. [1]): (1) как пространство коэффициентов унитарных представлений группы $G$ (функций вида $g \mapsto\langle\rho(g) \xi, \eta\rangle$, $\xi, \eta \in H_{\rho}$, где $H_{\rho}$ - пространство унитарного представления $\left.\rho\right)$; $(2)$ как пространство конечных линейных комбинаций положительно определенных функций на $G ;(3)$ как пространство непрерывных линейных функционалов на $C^{*}(G)$. Поскольку все это - пространства функций на группе $G$, то коммутативное умножение вводится поточечно.

ОПРЕДЕЛЕНИЕ 4.2. Идеал (Глимма) I называется обобщенной неподвижной точкой отображения $\hat{\phi}$, если линейная оболочка элементов $b-g[b]$ не плотна в 
$A_{I}=A / I$, т. е. ее замыкание $K_{I}$ не совпадает с $A_{I}$. Множество всех обобщенных неподвижных точек обозначим через GFP.

Если \# GFP < $<$, то скрученно-инвариантные РОА МАОП сосредоточены в этих точках. Для доказательства этого факта опишем действие группы $G$ на РОА МАОП более подробно. Ее действие на мерах определяется при помощи отождествления мер и функционалов на $A$.

Лемма 4.3. Если н отвечает скрученно-инвариантному функиионалу, то для любого борелевского подмножества $E \subset T$ функиионал $\mu(E)$ является скрученно-инвариантным.

ДокАзАтельство. Это немедленно следует из *-слабой регулярности. Действительно, пусть $a \in A, g \in G$ и $\varepsilon>0$ - сколь угодно малое число, а $U$ и $F$ определены в лемме 3.5 , причем для $a$ и $g[a]$ одновременно. Выберем такую непрерывную функцию $f: T \rightarrow[0,1]$, что $\left.f\right|_{F}=1$ и $\left.f\right|_{T \backslash U}=0$. Тогда

$$
\begin{aligned}
|\mu(E)(a-g[a])| & =|\mu(E \backslash F)(a)+\mu(F)(a)-\mu(E \backslash F)(g[a])-\mu(F)(g[a])| \\
& \leqslant|\mu(F)(a)-\mu(F)(g[a])|+2 \varepsilon=|\mu(F)(f a)-\mu(F)(g[f a])|+2 \varepsilon \\
& =|\mu(U)(f a)-\mu(U \backslash F)(f a)-\mu(U)(g[f a])+\mu(U \backslash F)(g[f a])|+2 \varepsilon \\
& \leqslant|\mu(U)(f a)-\mu(U)(g[f a])|+4 \varepsilon \\
& =|\mu(T)(f a)-\mu(T)(g[f a])|+4 \varepsilon=4 \varepsilon .
\end{aligned}
$$

ЛЕмма 4.4. Для любого скрученно-инвариантного функиионала $\varphi$ на $C^{*}(G)$ соответствующая мера $\mu$ сосредоточена на множестве GFP обобщенных неподвижных точек.

ДокАЗАТЕЛЬСтво. Пусть $\|\mu\|=1$. Предположим противное: существует элемент $a \in A,\|a\|=1$, обращающийся в нуль на неподвижных точках, причем $\varphi(a) \neq 0$. Пусть $\varepsilon:=|\varphi(a)|>0$. Для каждой точки $t \notin \mathrm{GFP}$ мы можем найти такие элементы $b_{t}^{i} \in A, g_{t}^{i} \in G, i=1, \ldots, k(t)$, что

$$
\left\|a(t)-\sum_{i=1}^{k(t)}\left(g_{t}^{i}\left[b_{t}^{i}\right](t)-b_{t}^{i}(t)\right)\right\|<\frac{\varepsilon}{4} .
$$

Тогда найдется такая окрестность $U_{t}$ точки $t$, что для $s \in U_{t}$ выполнено неравенство

$$
\left\|a(s)-\sum_{i=1}^{k(t)}\left(g_{t}^{i}\left[b_{t}^{i}\right](s)-b_{t}^{i}(s)\right)\right\|<\frac{\varepsilon}{2} .
$$

Выберем конечное подпокрытие $\left\{U_{t_{j}}\right\}, j=1, \ldots, n$, из $\left\{U_{t}\right\}$ и борелевское разбиение $E_{1}, \ldots, E_{n}$, подчиненное этому подпокрытию. Тогда

$$
\begin{aligned}
\varphi(a) & =\sum_{j=1}^{n} \mu\left(E_{j}\right)(a) \\
& =\sum_{j=1}^{n} \mu\left(E_{j}\right)\left(a-\sum_{i=1}^{k\left(t_{j}\right)}\left(g_{t_{j}}^{i}\left[b_{t_{j}}^{i}\right]-b_{t_{j}}^{i}\right)\right)+\sum_{j=1}^{n} \sum_{i=1}^{k\left(t_{j}\right)} \mu\left(E_{j}\right)\left(g_{t_{j}}^{i}\left[b_{t_{j}}^{i}\right]-b_{t_{j}}^{i}\right) .
\end{aligned}
$$

По лемме 4.3 каждое слагаемое во втором члене равно нулю. Абсолютное значение первого члена не превосходит $\sum_{j}\left\|\mu\left(E_{j}\right)\right\| \varepsilon / 2 \leqslant\|\mu\| \cdot \varepsilon / 2=\varepsilon / 2$. Это противоречит тому, что $|\varphi(a)|=\varepsilon$. 
Поскольку функционал $\varphi$ на $A_{I}$ является скрученно-инвариантным тогда и только тогда, когда $\operatorname{Ker} \varphi \supset K_{I}$, то размерность пространства этих функционалов равняется размерности пространства функционалов на $A_{I} / K_{I}$ и конечна тогда и только тогда, когда пространство $A_{I} / K_{I}$ конечномерно. В этом случае размерность пространства скрученно-инвариантных функционалов на $A_{I}$ равняется $\operatorname{dim}\left(A_{I} / K_{I}\right)$.

ОПРЕДЕЛЕНИЕ 4.5. Обобщенное число $S_{*}(\phi)$ неподвижных точек $\hat{\phi}$ на спектре Глимма определяется так:

$$
S_{*}(\phi):=\sum_{I \in \mathrm{GFP}} \operatorname{dim}\left(A_{I} / K_{I}\right) .
$$

Поскольку функционалы, отвечающие мерам, сосредоточенным в различных точках, являются линейно независимыми (пространство хаусдорфово), то в силу проведенных рассуждений верна следующая теорема.

ТЕоремА 4.6 (слабая обобщенная типа Бернсайда). Если хотя бъ одно из чисел $R_{*}(\phi), S_{*}(\phi)$ конечно, то

$$
R_{*}(\phi)=S_{*}(\phi) .
$$

\section{ЛитерАТУРА}

1. Диксмъе Ж. $C^{*}$-алгебры и их представления. Мир, М., 1974.

2. Кириллов A. А. Элементы теории представлений. Наука, М., 1978.

3. Мерфи A. $C^{*}$-алгебры и теория операторов. Факториал Пресс, М., 1997.

4. Фелъштын A. Л. Число Райдемайстера любого автоморфизма гиперболической по Громову группы бесконечно. Зап. научн. сем. ПОМИ, 279, №6 (Геом. и топол.), 229-240, 250 (2001).

5. Arthur J., Clozel L. Simple Algebras, Base Change, and the Advanced Theory of the Trace Formula. Princeton University Press, Princeton, NJ, 1989.

6. Dauns J., Hofmann K. H. Representations of rings by continuous sections. Mem. Amer. Math. Soc., Vol. 83, Amer. Math. Soc., Providence, RI, 1968.

7. Fell J. M. G. The structure of algebras of operator fields. Acta Math., 106, 233-280, 1961.

8. Fel'shtyn A. Dynamical zeta functions, Nielsen theory and Reidemeister torsion. Mem. Amer. Math. Soc., 147, No. 699, 2000.

9. Fel'shtyn A., Troitsky E. A twisted Burnside theorem for countable groups and Reidemeister numbers. In: Proc. Workshop Noncommutative Geometry and Number Theory (Bonn, 2003, Consani K., Marcolli M., Manin Yu., eds.), Vieweg, Braunschweig, 2006, pp. 141-154; Preprint MPIM2004-65; работа принята в Math. Res. Lett.

10. Fel'shtyn A., Troitsky E., Vershik A. Twisted Burnside theorem for type $\mathrm{II}_{1}$ groups: an example. Preprint 85, Max-Planck-Institut für Mathematik, 2004.

11. Grothendieck A. Formules de Nielsen-Wecken et de Lefschetz en géométrie algébrique. In: Séminaire de Géométrie Algébrique du Bois-Marie 1965-66. SGA 5, Lecture Notes in Math., Vol. 569, Springer-Verlag, Berlin, 1977, pp. 407-441.

12. Jiang B. Lectures on Nielsen Fixed Point Theory, Contemp. Math., Vol. 14, Amer. Math. Soc., Providence, RI, 1983.

13. Pedersen G. K. $C^{*}$-Algebras and Their Automorphism Groups. Academic Press, London-New York-San Francisco, 1979.

14. Shokranian S. The Selberg-Arthur Trace Formula. Based on lectures by James Arthur, Springer-Verlag, Berlin, 1992. 